\title{
Generalized quaternion M sets and Julia sets perturbed by dynamical noises
}

\author{
Yuanyuan Sun · Peng Li · Zhixing Lu
}

Received: 23 August 2014 / Accepted: 5 May 2015 / Published online: 24 May 2015

(C) The Author(s) 2015. This article is published with open access at Springerlink.com

\begin{abstract}
In this study, the quaternion Mandelbrot sets and Julia sets (abbreviated as M-J sets) with additive and multiplicative noise perturbations are constructed and the changes of their fractal characteristics are explored. The experimental results show that the quaternion $\mathrm{M}$ sets with additive noise perturbations move in the direction of the noise, while the structures remain unchanged. The quaternion $\mathrm{J}$ sets with additive noise perturbations change dramatically both in the structures and in the periodicities. The quaternion $\mathrm{M}$ sets with multiplicative noise perturbations present scaling and rotation, while the stable regions maintain the same distributions as the non-perturbed $\mathrm{M}$ sets. The structures and the periodicities of the $\mathrm{J}$ sets change under multiplicative noise perturbations, keeping different sensitivity to the noise parameters. The $\mathrm{M}$ sets and $\mathrm{J}$ sets still share the same stable points under both the additive and multiplicative noise perturbations.
\end{abstract}

Keywords Generalized M sets · Generalized J sets · Noise perturbations · Quaternion · Fractal

\section{Introduction}

Mandelbrot sets and Julia sets are the classic sets in the fractal geometries. The researches on the M-J sets

Y. Sun $(\varangle) \cdot$ P. Li · Z. Lu

College of Computer Science and Technology, Dalian

University of Technology, Dalian 116023, China

e-mail: syuan@dlut.edu.cn focus on the discussion of the structure stability of the iterative dynamic systems. In recent years, the highdimensional fractals have drawn people's much attention. The quaternion $\mathrm{M}-\mathrm{J}$ sets are one of the major areas people interested in, researches which help explore the 3 -space dynamics [1-5]. The subject of dynamics is concerned with what happens to a physical or geometric system over time, when it is subjected to a force or undergoes some kind of manipulation. The dynamics of motion in 3-space is easily expressed in terms of quaternion operation. This relationship implies computational advantages in using quaternions to express 3-D spatial manipulation [1].

On the other hand, there is a variety of noise disturbance in nature. They produce various effects on the movements and the states of the dynamic systems. The noise is a random variable essentially. People utilized different models, such as Gaussian noise [6], sinusoidal noise [6] and time-delay model [7], to simulate the interference characteristics of the dynamic system evolution.

Argyris et al. [8-10] analyzed the classification and influence of noise in a dynamical system and proposed a new scheme of analytic and non-analytic perturbations of the Mandelbrot map. Andreadis and Karakasidis [11-13] also discussed the topological closeness of perturbed M-J sets and proposed a definition for a probabilistic Mandelbrot map. Negi et al. introduced a new noise criterion and analyzed its effect on superior $\mathrm{M}-\mathrm{J}$ maps, discussing the difference comparing the usual M-J maps with perturbation [14-16]. Rani 
and Agarwal [17] presented an integrated approach to study the additive and multiplicative noises with respect to perturbations in superior Julia sets. Wang et al. $[18,19]$ researched on the structural characteristic and the fission-evolution law of perturbed generalized M-J sets on complex plane. Sun and Wang [20] analyzed the effect of noise perturbation on the quaternion M sets. Eliazar [21] laid more focus on the noise in relationship between noise and fractal, showing that classic shot noise is intrinsically fractal. On the other hand, the M-J sets of the other models are proposed and some non-trivial results are obtained. Wang and Song [22] studied the generalized M-J sets for bicomplex numbers. Liu [23] studied the M sets in coupled map lattice for control and synchronization. Danca et al. [24] obtained the connectivity domains of alternated Julia sets, defined by switching the dynamics of two quadratic Julia sets.

In this paper, the generalized quaternion $\mathrm{M}-\mathrm{J}$ sets perturbed with dynamical noise are studied. The quaternion $\mathrm{M}-\mathrm{J}$ sets are constructed, and the additive and multiplicative noise perturbations are interfered on the sets. The topological structures, the stable regions and the periodicities of the disturbed $\mathrm{M}-\mathrm{J}$ sets are explored. The rest of the paper is organized as follows. Section 1 introduces the definitions of the generalized quaternion $\mathrm{M}-\mathrm{J}$ sets under dynamical noise perturbations. Section 2 describes the influence on the quaternion $\mathrm{M}-\mathrm{J}$ sets with additive dynamic noises. The displacement of the stability regions of the $\mathrm{M}$ sets is theoretically analyzed, the characterization of the $\mathbf{J}$ sets is discussed, and the stable periodic points of the quaternion $\mathrm{M}-$ $\mathbf{J}$ sets are calculated. Section 3 discusses the influence on the quaternion $\mathrm{M}-\mathrm{J}$ sets with multiplicative dynamic noises. The symmetry of the $\mathrm{M}$ sets is proved, the 1cycle region boundary of one $\mathrm{M}$ set is calculated, and the structure changes of the $\mathrm{J}$ sets are discussed. Section 4 is the conclusion of the study.

\section{Noise perturbations in dynamical systems}

\subsection{The form of the dynamical noise}

The nature of the fluctuations introduced into a dynamical model depends on the coupling between the physical process and the source of random perturbations [25]. When a noise interferes with the evolution of a dynamical system, it is called a dynamical noise. Such dynamical noise may take the form of an additive or multiplicative expression which illustrates the kind of parameters by which noise may appear in the equations of a dynamical system.

The analog of additive noise is of the following form,

$x_{n+1}=f\left(\lambda, x_{n}\right)+p_{n}$

where $p_{n}$ represents a random deviation from the deterministic orbit.

The multiplicative dynamical noise perturbs the form of the nonlinear function or the parameters in the map. If we write the deterministic equation as

$x_{n+1}=\lambda f\left(x_{n}\right)$

with $\lambda$ as the bifurcation parameter, then parametric fluctuations would be of the form

$x_{n+1}=\left(\lambda+q_{n}\right) f\left(x_{n}\right)$

with $q_{n}$ representing the fluctuations in the parameter $\lambda[26]$.

2.2 Noise perturbations of the generalized quaternion $\mathrm{M}-\mathrm{J}$ sets

When the generalized $\mathrm{M}$ sets are perturbed by additive noise, the iterate function is as follows:

$Q_{n+1}=Q_{n}^{\alpha}+c+m$

where $Q, c, m \in \mathbf{H}, \alpha \in \mathbf{N}, Q=S+\mathbf{i} \sigma \cdot V, c=$ $a+\mathbf{i} \sigma \cdot K, m=m_{1}+\mathbf{i} \sigma \cdot m^{\prime} . m$ is the noise variable, $m^{\prime}=\frac{m_{2} \mathbf{i}+m_{3} \mathbf{j}+m_{4} \mathbf{k}}{\sqrt{m_{2}^{2}+m_{3}^{2}+m_{4}^{2}}}$, and $m_{i} \in \mathbf{R}(i=1,2,3,4)$ are the parameters specifying the intensity of noise.

The generalized quaternion $\mathrm{M}$ set with the multiplicative dynamic noise is given by

$Q_{n+1}=(1+k) Q_{n}^{\alpha}+c$

where $k=\left(k_{0}, k_{1}, k_{2}, k_{3}\right)\left(k_{i} \in \mathbf{R}, i=0,1,2,3\right)$ are the parameters specifying the intensity of noise.

The quaternion $\mathrm{J}$ sets with noise perturbations have the same iteration processes with $\mathrm{M}$ sets except that $c$ is a constant in the mapping. 


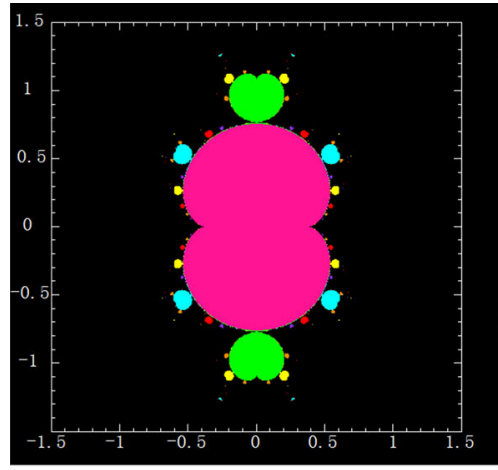

(a)

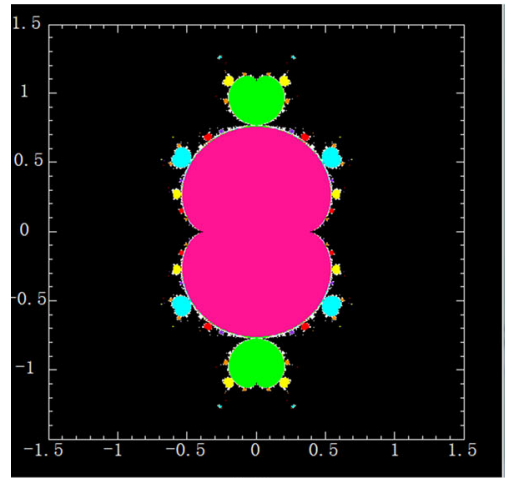

(d)

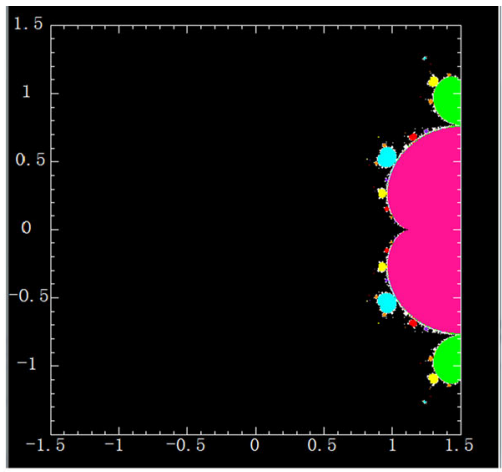

(b)

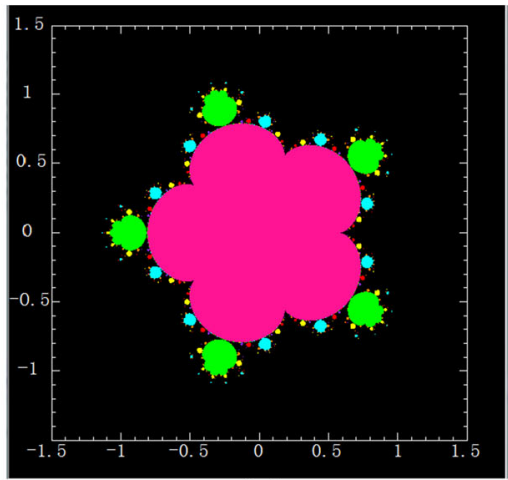

(e)

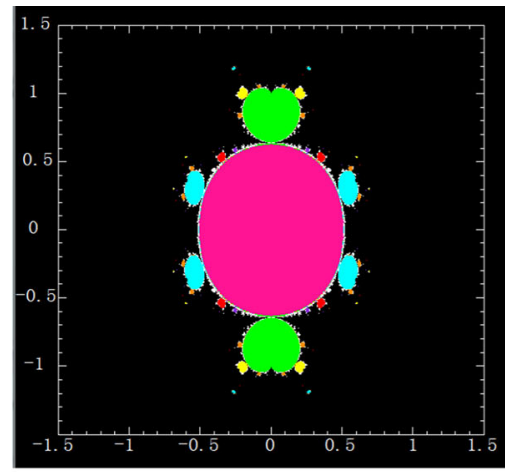

(c)

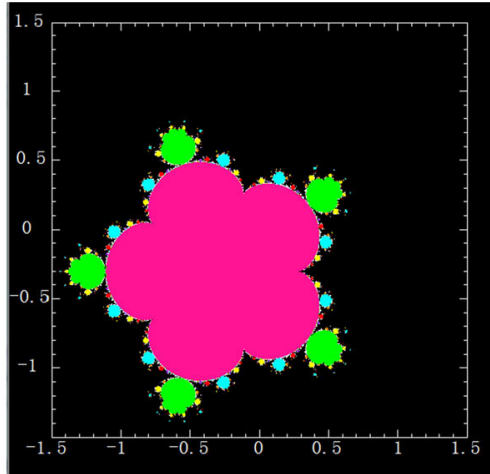

(f)
Fig. 1 Quaternion $\mathrm{M}$ sets with additive noise perturbations. $\mathbf{a} \alpha=3, m=(0,0,0,0), \mathbf{j}=\mathbf{k}=0$. b $\alpha=3, m=$ $(-1.5,0,0,0), \mathbf{j}=\mathbf{k}=0$. $\mathbf{c} \alpha=3, m=(0,0,0.3,0.3), \mathbf{j}=$

\section{Quaternion M-J sets with additive dynamic noises}

\subsection{Quaternion $\mathrm{M}$ sets with additive noises}

We utilize the cycle detecting method [27] to display the two-dimensional projection of the quaternion generalized $\mathrm{M}$ set. In this method, different cycle regions can be distinguished by different colors. The 1-cycle region is marked in pink, 2-cycle in green, 3-cycle in cyan, 4-cycle in yellow, 5-cycle in red, 6-cycle in orange, 7cycle in purple, and the escaped region in black. Almost all the figures in this paper are the projections on the real axis $\mathbf{r}$ and image axis $\mathbf{i}$, with the values of other two image axes $\mathbf{j}, \mathbf{k}$ being zero, except the ones with extra explanations. Figure 1 shows the quaternion $\mathrm{M}$ sets perturbed by additive noise $m$ with $\alpha=3$ and $\alpha=6$. Comparing these figures, we can find the struc- $\mathbf{k}=0 . \mathbf{d} \alpha=3, m=(0,0,0.3,0.3), \mathbf{j}=\mathbf{k}=-0.3$. $\mathbf{e} \alpha=6, m=(0,0,0,0), \mathbf{j}=\mathbf{k}=0$. f $\alpha=6, m=$ $(0.3,0.3,0,0), \mathbf{j}=\mathbf{k}=0$

tural changes with the interference of the additive noise $m$.

Figure 1a shows the original quaternion $\mathrm{M}$ set $(\alpha=$ 3 ) without noise perturbation, which displays consistent fractal structure with the $\mathrm{M}$ set in the complex plane. In Fig. 1b, we add the additive noise $m$ along the real axis $\mathbf{r}$. The results show that the whole image takes a translation along the reverse direction of the vector $m$, fractal structures remaining unchanged and the moving distance being $m$. In Fig. 1c, we add the additive noise $m$ along the image axis $\mathbf{j}$ and image axis $\mathbf{k}$. It shows that these projections change. To verify whether $\mathrm{M}$ set maintains translation only, we change our observation viewpoint. Taking the projection at $-m$, as shown in Fig. 1d, we can find the same topology structures with the original M set (Fig. 1a). Fig. 1e, f shows slice images of the quaternion $\mathrm{M}$ set $(\alpha=6)$ by the additive noise disturbance, showing the same phenomenon. 
Fig. 2 3-D projections of quaternion $\mathrm{M}$ sets with additive noise perturbations. a $\alpha=6, m=(0,0,0,0)$. $\mathbf{b}$ $\alpha=6, m=(0.4,0,0,0)$. c $\alpha=6, m=$

$(0.4,0,0.6,0.6)$

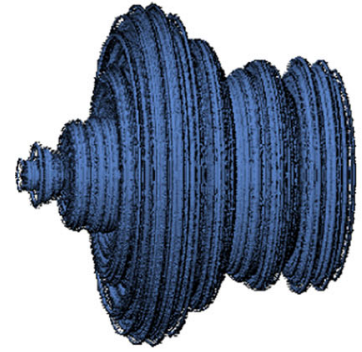

(a)

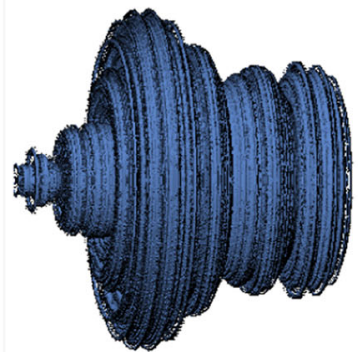

(b)

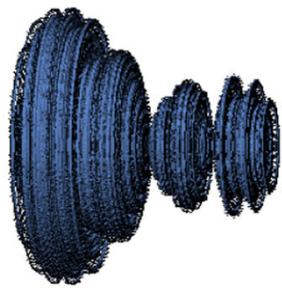

(c)

Now we give the following theorem.

Theorem 1 The stability regions of the quaternion $M$ sets with the additive dynamic noise $m$ move along the direction of $m$.

Proof The quaternion M set without noise perturbed is given by the mapping $F: z \leftarrow z^{\alpha}+c$. And for the noise case, the mapping is $f: z \leftarrow z^{\alpha}+c+m$. Now we explore the evolution of the iterations, and we can get $f^{(0)}(c)=c+m$. Let $c^{\prime}=c+m$, and then $f^{(0)}(c)=F^{(0)}\left(c^{\prime}\right)$. Similarly,

$f^{(1)}(c)=(c+m)^{\alpha}+c+m=c^{\prime \alpha}+c^{\prime}=F^{(1)}\left(c^{\prime}\right)$.

Suppose $f^{(n)}(c)=F^{(n)}\left(c^{\prime}\right)$ after $n$ iterations, and then after $n+1$ iterations

$$
\begin{aligned}
f^{(n+1)}(c) & =\left(f^{(n)}(c)\right)^{\alpha}+c+m \\
& =\left(F^{(n)}\left(c^{\prime}\right)\right)^{\alpha}+c^{\prime}=F^{(n+1)}\left(c^{\prime}\right) .
\end{aligned}
$$

The proof is completed.

\subsection{Stability regions of the $\mathrm{M}$ sets}

We calculate the 1-cycle region of the $\mathrm{M}$ sets with additive dynamic noises (see the detail in Appendix B). The boundary of the 1-cycle stability region $(\alpha=3)$ satisfies

$$
\begin{aligned}
a & =\frac{\sqrt{3}}{3} \cos \theta-\frac{\sqrt{3}}{9} \cos 3 \theta-m_{1}, \\
K & =\left(\frac{\sqrt{3}}{3} \sin \theta-\frac{\sqrt{3}}{9} \sin 3 \theta\right) n-m^{\prime}(0 \leq \theta<2 \pi) .
\end{aligned}
$$

Equation (3.1) shows that 1-cycle region moves along the direction of the noise $m$.

Corollary 1 The dynamics of the quaternion $M$ sets with additive dynamic noise on the mapping $f: z \leftarrow$ $z^{\alpha}+c+m$ is consistent with that of the mapping $F_{z+m}$ : $z \leftarrow z^{\alpha}+c$.

Corollary 1 indicates that quaternion $M$ sets with additive dynamic noise share the same properties with the original one, e.g., the former are symmetric around the point $m$ while the latter is around the origin [27]. As is shown in Fig. 2, we use escape time algorithm and ray-tracing method to construct the three-dimensional projections of the generalized M sets. In these figures, it can be more intuitive to observe the symmetry of the $\mathrm{M}$ sets with additive noise disturbance. Moreover, we may find the M sets' overall translational motions under additive noise perturbations and the different rendering with different viewpoints.

\subsection{Quaternion J sets with additive noises}

Figure 3 shows the quaternion $\mathrm{J}$ sets $(\alpha=5)$ disturbed by different additive noises. These slice images are the projections on the real axis $\mathbf{r}$ and image axis $\mathbf{i}$, with the values of the other two image axes $\mathbf{j}$, $\mathbf{k}$ being zero. Similar to the M sets, different stable regions of the $\mathrm{J}$ sets are marked in different colors and the escaped region is marked in black.

It can be seen from Fig. 3 that the $\mathrm{J}$ sets are disturbed by different intensity noises; their periodities and the topology structures change dramatically. On the other hand, comparing Fig. 3c, e, we find the same experimental results of different $\mathrm{J}$ sets with different intensity of noise disturbance. Now we give the theorems as follows.

Theorem 2 The quaternion $J$ set on the mapping $F$ : $z \leftarrow z^{\alpha}+c$, if interfered by the additive noise $m$, keeps the same topological structure with the quaternion $J$ set on the mapping $f: z \leftarrow z^{\alpha}+c^{\prime}+m\left(c=c^{\prime}+m\right)$.

As we know, the $\mathrm{M}$ set is the parameter set of the $\mathrm{J}$ set without noise disturbance. In the complex plane, each point in the $\mathbf{M}$ set maps out a $\mathbf{J}$ set. That is, each 


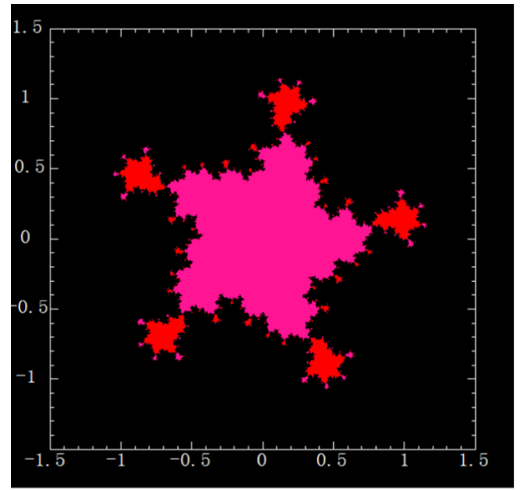

(a)

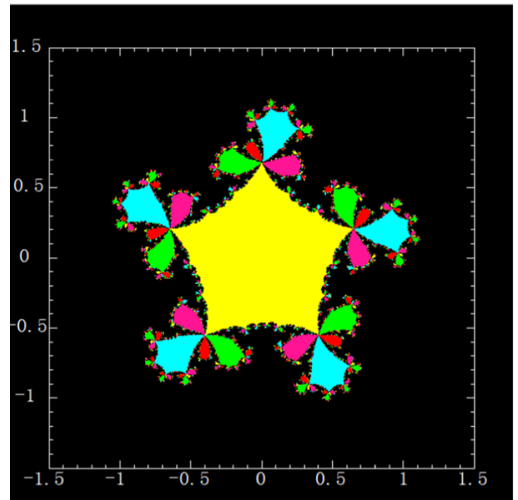

(d)

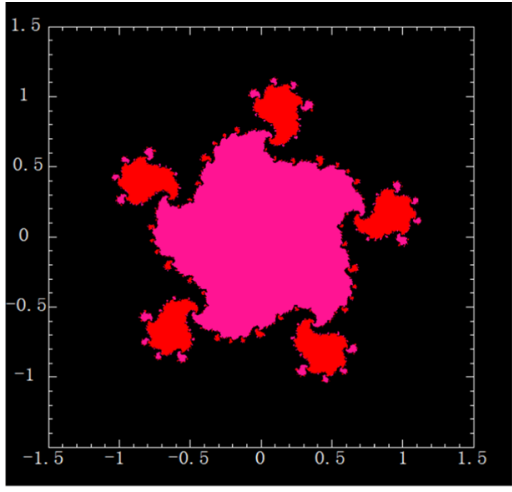

(b)

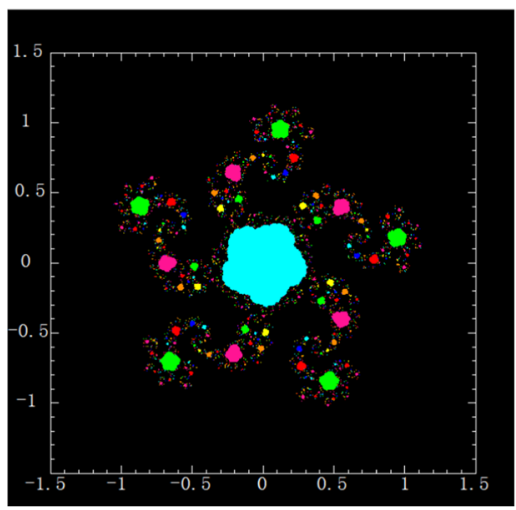

(e)

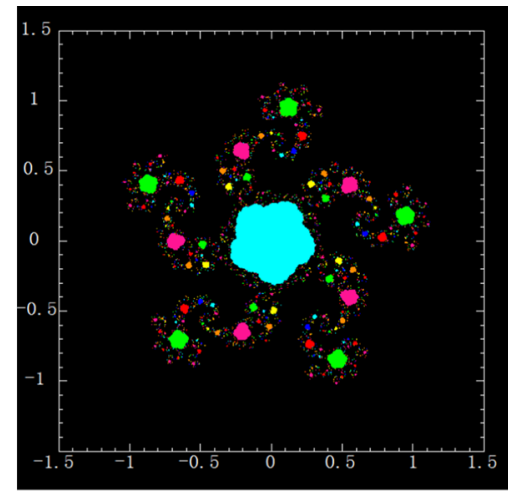

(c)

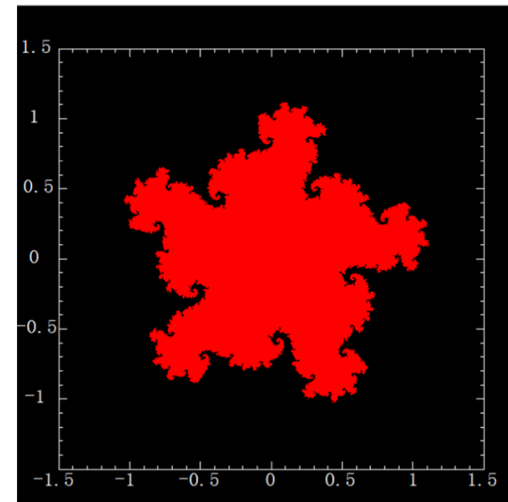

(f)
Fig. 3 Quaternion $\mathrm{J}$ sets with additive noise perturbations. $\mathbf{a} c=(-0.8,-0.7,0,0), m=(0,0,0,0)$. b $c=(-0.8,-0.7$, $0,0), m=(0.25,0,0,0)$. c $c=(-0.8,-0.7,0,0), m=$

$\mathrm{J}$ set only corresponds to a single point of the M set. However, it is shown in Theorem 2 that a $\mathbf{J}$ set can be mapped to all the points of the $\mathrm{M}$ sets if interfered by certain intensity additive noise.

Theorem 3 The generalized quaternion $J$ sets on the mapping $f: z \leftarrow z^{\alpha}+c+m$ can be described only by two variables of $c$, the scalar part $\left(a+m_{1}\right)$ and the module $\|c+m\|$.

The quaternion $\mathrm{J}$ set on the mapping $F: z \leftarrow z^{\alpha}+c$ is completely defined by the scalar part $a$ and $\|c\|$ [27]. It is easy to check that it also applies to the generalized quaternion $\mathrm{J}$ sets with additive noise perturbations.

Now we can observe the structure changes of the quaternion $\mathbf{J}$ set with the additive noise disturbance from the three-dimensional perspective view (see Fig. 4).
$(0.3,0,0,0)$. d $c=(-0.4,-0.7,0,0), m=(0,0,0,0)$. e $c=(-0.4,-0.7,0,0), m=(-0.1,0,0,0)$. f $c=$ $(-0.4,-0.7,0,0), m=(-0.1,0.1,0.1,0.1)$

\subsection{The stable periodic points of the quaternion $\mathrm{M}-\mathrm{J}$ sets}

It is known that the $\mathrm{M}$ set and $\mathrm{J}$ set on the same mapping reflect the same dynamic system. $M$ set is the illustration directory of $\mathbf{J}$ set. The experimental results in Sects. 3.1 and 3.2 show that under the additive noise disturbance the $\mathrm{M}$ sets present only simple displacement, while the $\mathrm{J}$ sets have dramatic changes on the periodicities and the topological structures. To verify whether $\mathrm{M}-\mathrm{J}$ sets still retain the mapping relationship under the disturbances, we calculate several groups of the stable periodic points in $\mathbf{M}-\mathrm{J}$ sets under the same intensity noise disturbances. It is shown in Table 1 that quaternion $\mathbf{M}$ sets and $\mathbf{J}$ sets still share the same stable points under the additive noise perturbations. 
Fig. 4 3-D projections of quaternion $\mathrm{J}$ sets with additive noise perturbations. a $c=$ $(-0.9166,0.5635,0,0))$, $m=(0.2,0,0,0) . \mathbf{b} c=$ $(-0.9166,0.5635,0,0))$, $m=(0.4,0,0,0) . \mathbf{c} c=$ $(-0.9166,0.5635,0,0))$, $m=(0.2,-0.05,0,0)$

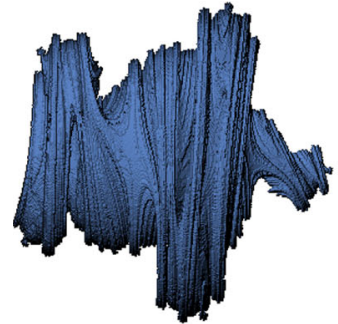

(a)

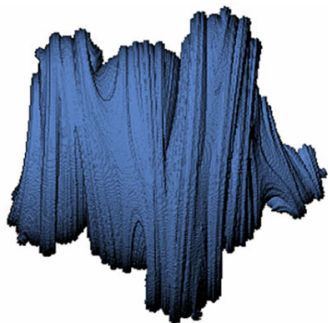

(b)

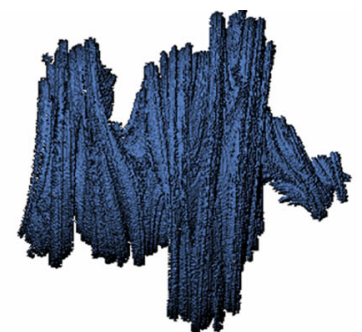

(c)

Table 1 Stable periodic points in M-J sets with additive noise perturbations $(c=(-0.4,-0.7,0.01,-0.02))$

\begin{tabular}{lll}
\hline$m=(0,0,0,0)$ & $m=(0.01,0.02,0,0)$ & $m=(0.01,0.02,-0.02,0.03)$ \\
\hline M sets & & \\
$(-0.4000,-0.7000,0.0100,-0.0200)$ & $(-0.3809,-0.6747,0.0099,-0.0198)$ & $(-0.3806,-0.6744,-0.0099,0.0099)$ \\
$(-0.5775,-0.4087,0.0058,-0.0117)$ & $(-0.5417,-0.4450,0.0065,-0.0131)$ & $(-0.5412,-0.4459,-0.0066,0.0066)$ \\
$(-0.2230,-0.7109,0.0102,-0.0203)$ & $(-0.2279,-0.6302,0.0093,-0.0185)$ & $(-0.2282,-0.6291,-0.0093,0.0093)$ \\
$(-0.6297,-0.7119,0.0102,-0.0203)$ & $(-0.5237,-0.6579,0.0097,-0.0194)$ & $(-0.5224,-0.6574,-0.0097,0.0097)$ \\
$(-0.0425,-0.0107,0.0002,-0.0003)$ & $(-0.2986,-0.2690,0.0040,-0.0079)$ & $(-0.3007,-0.2719,-0.0040,0.0040)$ \\
$J$ sets & & \\
$(-0.4000,-0.7000,0.0100,-0.0200)$ & $(-0.3809,-0.6747,0.0099,-0.0198)$ & $(-0.3806,-0.6744,-0.0099,0.0099)$ \\
$(-0.5775,-0.4087,0.0058,-0.0117)$ & $(-0.5417,-0.4450,0.0065,-0.0131)$ & $(-0.5412,-0.4459,-0.0066,0.0066)$ \\
$(-0.2230,-0.7109,0.0102,-0.0203)$ & $(-0.2279,-0.6302,0.0093,-0.0185)$ & $(-0.2282,-0.6291,-0.0093,0.0093)$ \\
$(-0.6297,-0.7119,0.0102,-0.0203)$ & $(-0.5237,-0.6579,0.0097,-0.0194)$ & $(-0.5224,-0.6574,-0.0097,0.0097)$ \\
$(-0.0425,-0.0107,0.0002,-0.0003)$ & $(-0.2986,-0.2690,0.0040,-0.0079)$ & $(-0.3007,-0.2719,-0.0040,0.0040)$
\end{tabular}

\section{Quaternion M-J sets with multiplicative noises}

\subsection{Quaternion M sets with multiplicative noises}

Figure 5 shows the quaternion $\mathrm{M}$ sets with $\alpha=3$ disturbed by multiplicative noises $k=\left(k_{0}, k_{1}, k_{2}, k_{3}\right)$ ( $\left.k_{i} \in \mathbf{R}, i=0,1,2,3\right)$. The colors marking different stable regions are the same with that of the $\mathrm{M}$ sets in Sect. 3. Figure 5b, c shows the M sets with the multiplicative noise perturbations on the real axis $\mathbf{r}$. When the noise intensity is enhanced continuously, each cycle region is longitudinally compressed, separated with each other and the whole area of the M sets contracting. Figure 5d, e shows the $\mathrm{M}$ sets under the multiplicative noise perturbations from the image axis $\mathbf{i}$. When the noise intensity is enhanced, the cycle regions are transversely compressed and the nested structures present at the boundary of the adjacent cycle regions. Figure $5 \mathrm{f}$ shows the area of the $\mathrm{M}$ set contracts in a certain proportion compared with the non-perturbation M set.
It is found that the $\mathrm{M}$ sets are still symmetric around the real axis under multiplicative noise perturbations, which is different from the displacement changes caused by additive noise. Now we give the following theorem and prove it theoretically.

Theorem 4 If the quaternion $q\left(q_{0}, q_{1}, q_{2}, q_{3}\right)\left(q_{i} \in\right.$ $R, i=0,1,2,3)$ is in the quaternion $M$ set with the multiplicative dynamic noise perturbed, supposing $q_{1}^{2}+q_{2}^{2}+q_{3}^{2}=\rho^{2}(\rho>0)$, then any quaternion $q^{\prime}\left(q_{0}^{\prime}, q_{1}^{\prime}, q_{2}^{\prime}, q_{3}^{\prime}\right)\left(q_{i}^{\prime} \in R, i=0,1,2,3\right)$ that satisfies $q_{0}=q_{0}^{\prime}$ and $q_{1}^{\prime 2}+q_{2}^{\prime 2}+q_{3}^{\prime 2}=\rho^{2}$ is also in the M sets.

Proof We prove it by mathematical induction. According to Eq. (2.5), after the first iteration it becomes

$$
\begin{aligned}
z^{(1)}= & \left(1+k_{1}\right) h^{\alpha} \cos \alpha \theta+h \cos \theta \\
& +\left[\left(1+k_{2}\right) h^{\alpha} \sin \alpha \theta+h \sin \theta\right] n,
\end{aligned}
$$

where $n$ is unit axis of rotation and $n=\left(q_{1} i+q_{2} j+\right.$ $\left.q_{3} k\right) / \rho, h=\sqrt{q_{0}^{2}+\rho^{2}}, \theta=\arctan \frac{\rho}{q_{0}}$. 


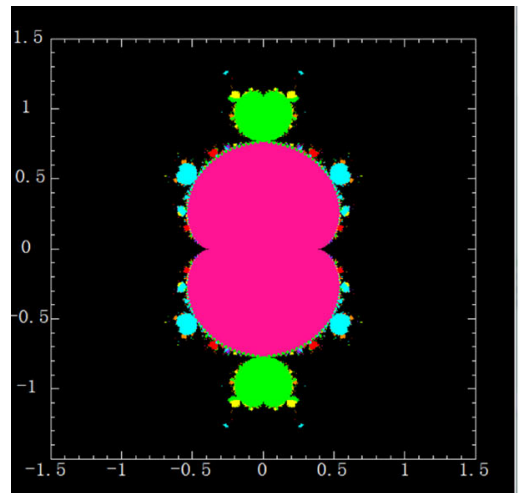

(a)

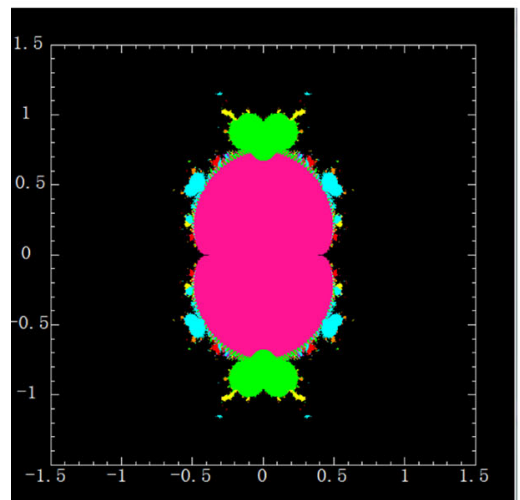

(d)

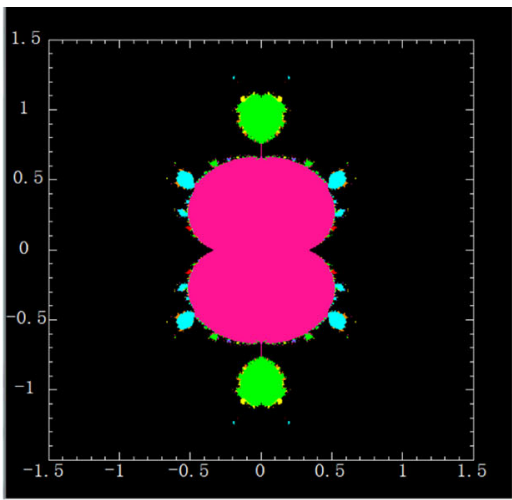

(b)

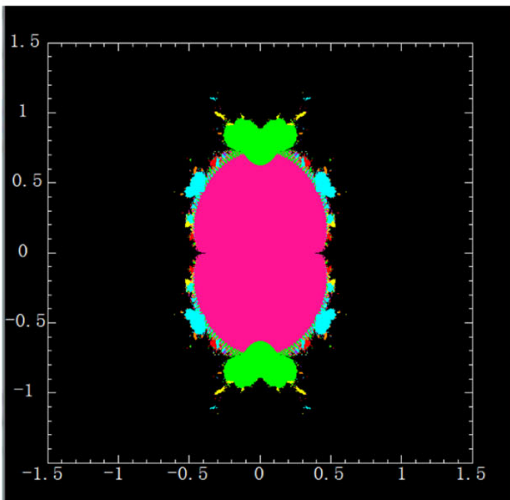

(e)

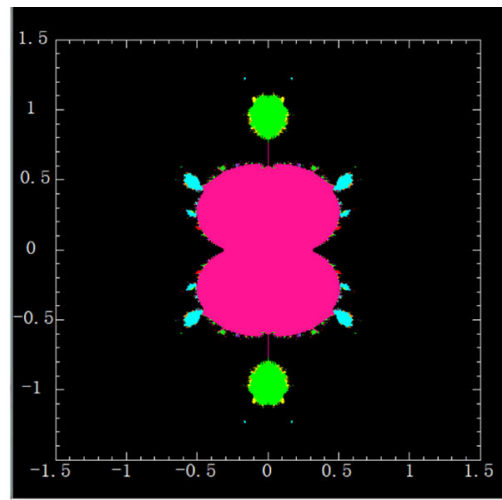

(c)

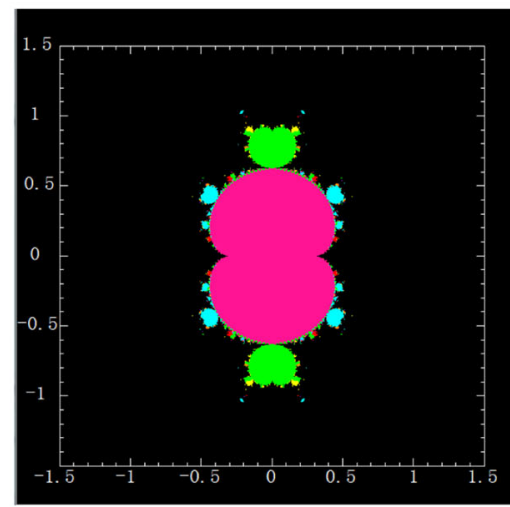

(f)

Fig. 5 Quaternion $M$ sets with multiplicative noise perturbations $(\alpha=3)$. a $k=(0,0,0,0)$. $\mathbf{b} k=(0.3,0,0,0)$. $\mathbf{c} k=(0.5,0,0,0)$. d $k=(0,0.3,0,0)$. e $k=(0,0.5,0,0)$. $\mathbf{f} k=(0.5,0.5,0,0)$

By analogy,

$$
\begin{aligned}
z^{\prime(1)}= & \left(1+k_{1}\right) h^{\prime \alpha} \cos \alpha \theta^{\prime}+h^{\prime} \cos \theta^{\prime} \\
& +\left[\left(1+k_{2}\right) h^{\prime \alpha} \sin \alpha \theta^{\prime}+h^{\prime} \sin \theta^{\prime}\right] n^{\prime},
\end{aligned}
$$

where $n^{\prime}$ is unit axis of rotation and $n^{\prime}=\left(q_{1}^{\prime} i+q_{2}^{\prime} j+\right.$ $\left.q_{3}^{\prime} k\right) / \rho, h^{\prime}=\sqrt{q_{0}^{\prime 2}+\rho^{2}}, \theta^{\prime}=\arctan \frac{\rho}{q_{0}^{\prime}}$.

Since $q_{0}=q_{0}^{\prime}, h=h^{\prime}, \theta=\theta^{\prime}$. From Eqs. (4.1) and (4.2), we have

$\rho^{(1)}=\left|\left(1+k_{2}\right) h^{\alpha} \sin \alpha \theta+h \sin \theta\right|=\rho^{\prime(1)}$.

Then we can get the properties as follows,

(1) $q_{0}^{(1)}=q_{0}^{\prime(1)}$

(2) $\rho^{(1)}=\rho^{\prime(1)}$

(3) The directions of the vectors of $z^{(1)}$ and $z_{1}^{\prime(1)}$ do not change after iterations, which are the same with $n$ and $n^{\prime}$, respectively.

Suppose $z^{(m)}$ and $z^{\prime(m)}$ retain the above properties after $m$ iterations, and then after $m+1$ iterations we have

$$
\begin{aligned}
z^{(m+1)}= & \left(1+k_{1}\right) h^{(m) \alpha} \cos \alpha \theta^{(m)}+h \cos \theta \\
& +\left[\left(1+k_{2}\right) h^{(m) \alpha} \sin \alpha \theta^{(m)}+h \sin \theta\right] n \\
z^{\prime(m+1)}= & \left(1+k_{1}\right) h^{\prime(m) \alpha} \cos \alpha \theta^{\prime(m)}+h \cos \theta \\
& +\left[\left(1+k_{2}\right) h^{\prime(m) \alpha} \sin \alpha \theta^{\prime(m)}+h \sin \theta\right] n^{\prime}
\end{aligned}
$$

From Eqs. (4.4) and (4.5), we conclude that $z^{(m+1)}$ and $z^{\prime(m+1)}$ satisfy the above three conditions by induction. Therefore, if $q \in \mathrm{M}, q^{\prime} \in \mathrm{M}$.

The proof is completed.

\subsection{Stability regions of the $\mathrm{M}$ sets}

The 1-cycle stability region of the $\mathrm{M}$ sets with multiple dynamic noise is calculated in Appendix C. The boundary of the 1-cycle stability region satisfies 


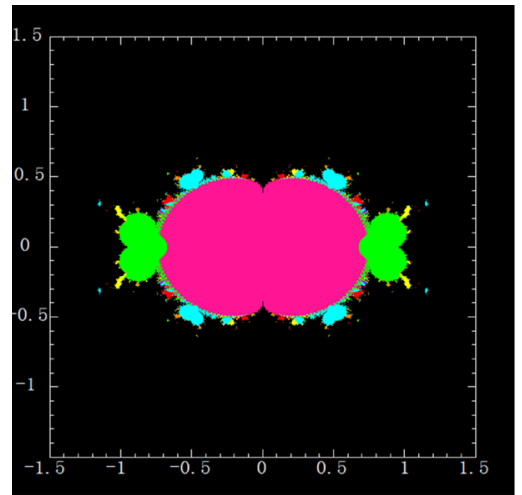

(a)

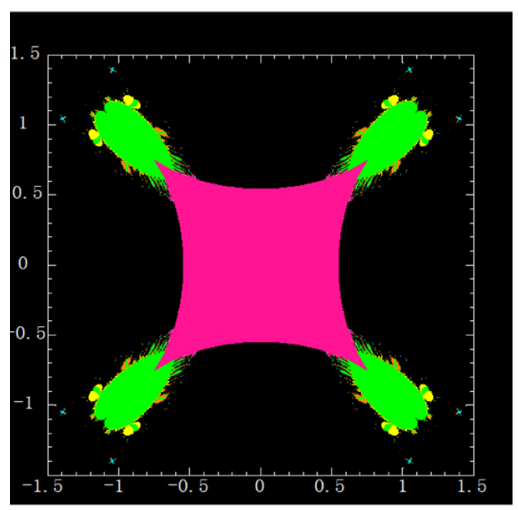

(d)

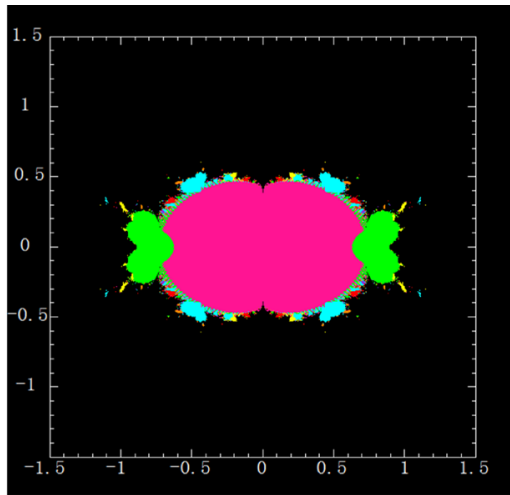

(b)

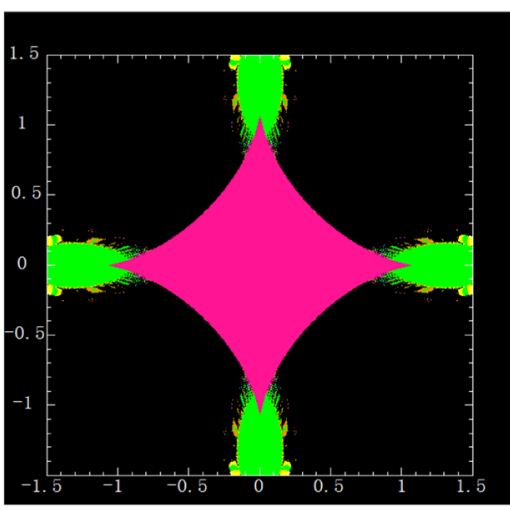

(e)

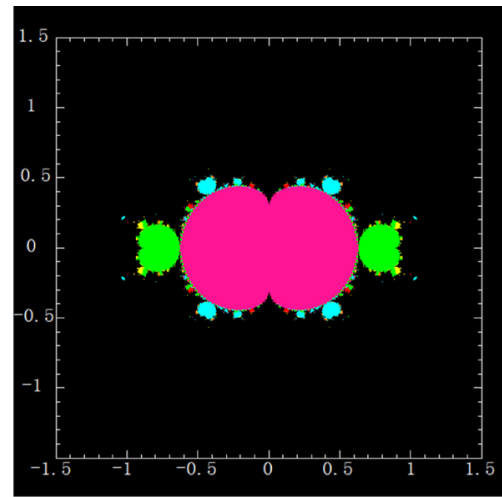

(c)

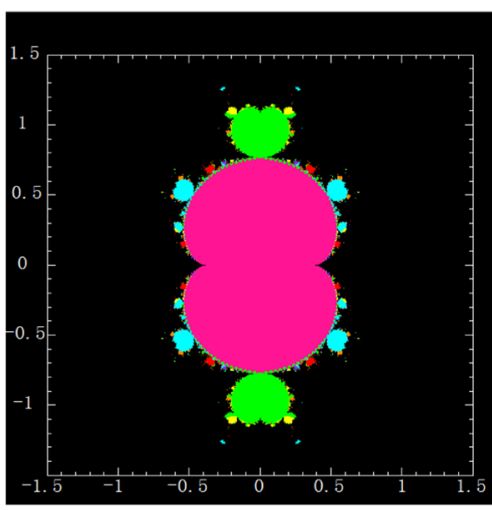

(f)

Fig. 6 Quaternion $M$ sets with multiplicative noise perturbations. a $\alpha=3, k=(-2.3,-2,0,0)$. $\mathbf{b} \alpha=3, k=(-2.5,-2,0,0)$. $\mathbf{c} \alpha=3, k=(-2.5,-2.5,0,0)$. d $\alpha=3, k=(-0.5,-1.5,0,0)$. e $\alpha=3, k=(-1.5,-0.5,0,0)$. f $\alpha=3, k=(0,0,3,3)$

$$
\begin{aligned}
a= & \left(\frac{1}{9 p q}\right)^{\frac{1}{4}} \cos \theta-\alpha\left(\frac{1}{9 p q}\right)^{\frac{3}{4}} \cos 3 \theta, \\
K= & {\left[\left(\frac{1}{9 p q}\right)^{\frac{1}{4}} \sin \theta-\beta\left(\frac{1}{9 p q}\right)^{\frac{3}{4}} \sin 3 \theta\right] } \\
& n(0 \leq \theta<2 \pi) .
\end{aligned}
$$

Equation (4.6) explains why the M sets in Fig. 5 shrink while keeping with some proportion.

To further analyze the impact of the changes of $m$ on the M sets, the value range of $m$ is extended to the whole four-dimensional space. The experimental results are shown in Fig. 6. When $k_{0}$ and $k_{1}$ are greater than -1 , the $\mathrm{M}$ sets appear the longitudinal and the transverse compressional deformation, which is the same with the results in Fig. 5. When $k_{0}$ is equal to $k_{1}$, the $\mathrm{M}$ sets render scaling morphology. And the areas of the M sets get larger with the value of $k_{0}$ closer to -1 . When $k_{0}$ and $k_{1}$ are less than -1 , the $\mathrm{M}$ sets flip with 90 degrees, comparing Fig. 6a-c with Fig. $5 d-$ f. When $k_{0}$ is greater than -1 and $k_{1}$ is less than -1 , "the Heart" (the pink area) inside the $\mathbf{M}$ set disappears and the projections on the axis $\mathbf{r}$ and $\mathbf{j}$ (or $\mathbf{k}$ ) present rhombus shape (see Fig. $6 \mathrm{~d}, \mathrm{e}$ ). When $k_{2}$ and $k_{3}$ are not zero, there is no influence on the projection image on the axis $\mathbf{r}$ and $\mathbf{j}$ (see Fig. 6f).

The three-dimensional projections of the quaternion $\mathrm{M}$ sets under the multiplicative noise disturbance are shown in Fig. 7.

\subsection{Quaternion J sets with multiplicative noises}

Figure 8 shows the multiplicative noise-perturbed quaternion $\mathrm{J}$ sets with $\alpha=5$. These slice images are the projections on the real axis $\mathbf{r}$ and image axis $\mathbf{i}$, the values of the other two image axes $\mathbf{j}, \mathbf{k}$ being zero. Different stable regions of the $\mathbf{J}$ sets are marked with 
Fig. 7 3-D projections of quaternion $\mathrm{M}$ sets with multiplicative noise perturbations. a $\alpha=3$, $k=(0,0,0,0) . \mathbf{b}$ $\alpha=3, k=(1,0,0,0)$. c $\alpha=3, k=(0,0,0.5,0.5)$

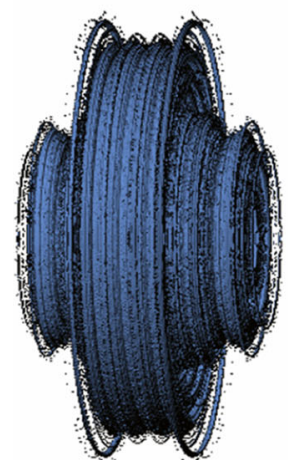

(a)

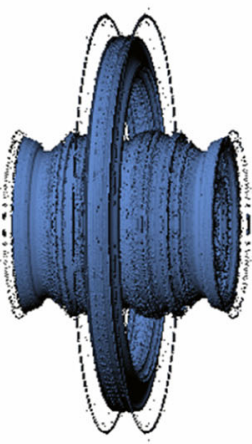

(b)

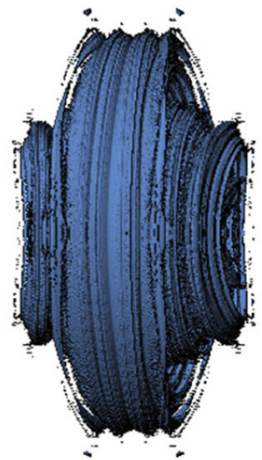

(c)

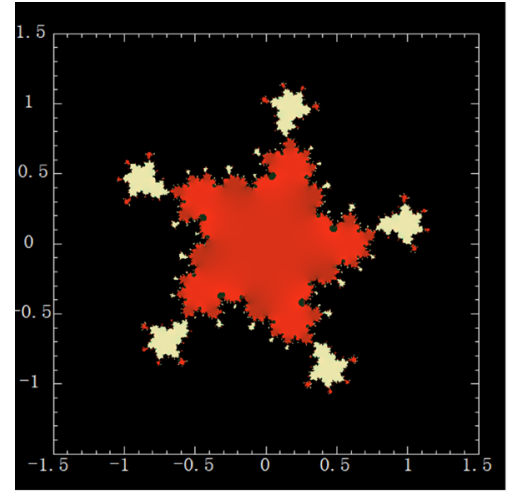

(a)

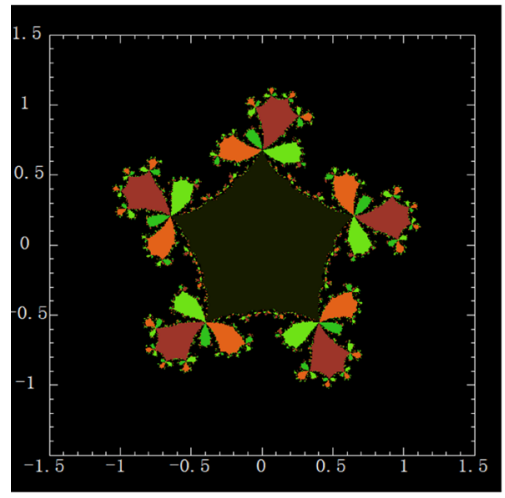

(d)

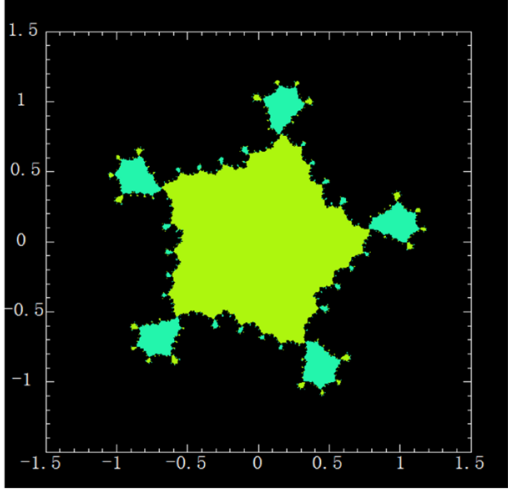

(b)

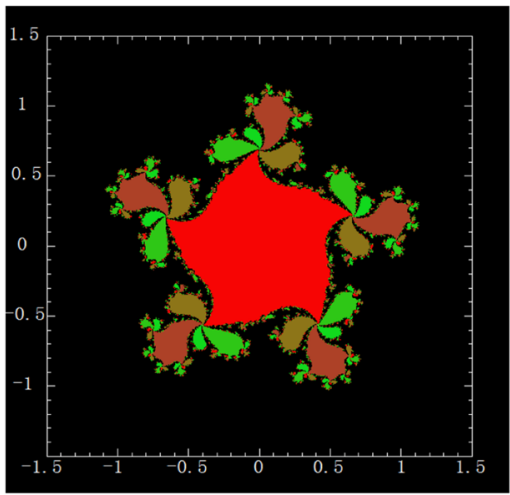

(e)

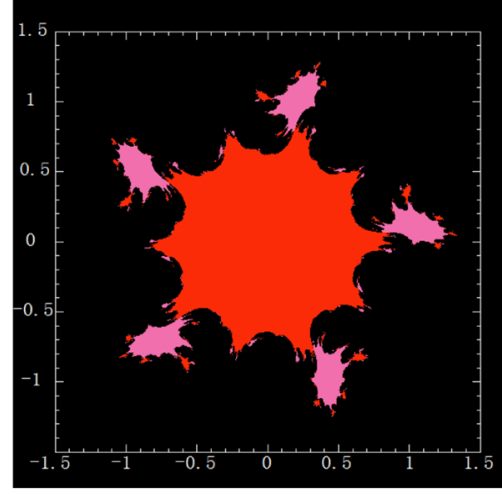

(c)

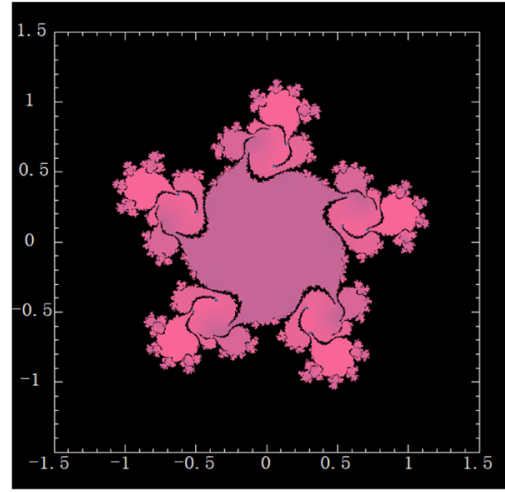

(f)

Fig. 8 Quaternion J sets with multiplicative noise perturbations. a $c=(-0.8,-0.7,0,0), m=(0.01,0,0,0)$. b $c=(-0.8$, $-0.7,0,0), m=(-0.1,0,0,0) . \mathbf{c} c=(-0.8,-0.7,0,0), m=$

different colors, and the escaped regions are marked with black.

Experimental results show that quaternion $\mathrm{J}$ sets with multiplicative noise disturbance have various changes in the topology structures and periodicities

$(-0.5,0,0,0)$. d $c=(-0.4,-0.7,0,0), m=(0,0,0,0)$. e $c=(-0.4,-0.7,0,0), m=(0,-0.12,0,0)$. f $c=$ $(-0.4,-0.7,0,0), m=(-0.12,-0.12,0,0)$

(see Fig. 8). Compared with the J sets, under additive noise disturbances they have a higher sensitivity to the multiplicative noises, which indicates the points escape faster. On the other hand, the number and the distribution of the buds on the $\mathrm{J}$ set boundary are not 
Fig. 9 3-D projections of quaternion $\mathrm{J}$ sets with multiplicative noise perturbations. a $c=(-0.8,-0.7,0,0)$, $m=(0,0,0,0) . \mathbf{b} c=$ $(-0.8,-0.7,0,0), m=$ $(-0.1,0,0,0) . \mathbf{c}$ $c=(-0.8,-0.7,0,0)$, $m=(-0.5,0,0,0)$

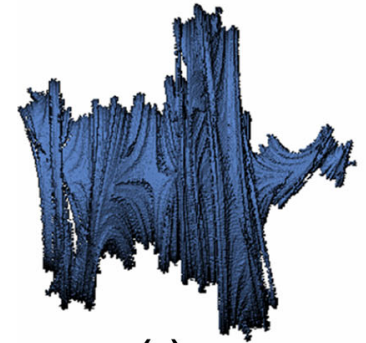

(a)

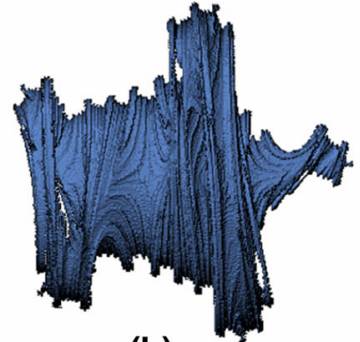

(b)

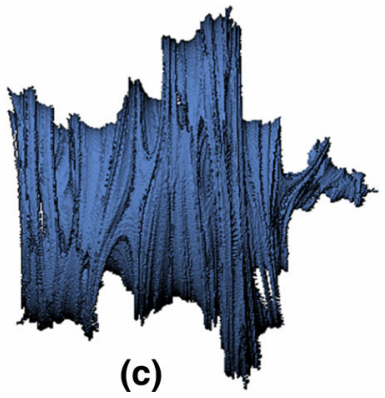

Table 2 Stable periodic points in M-J sets with additive noise perturbations $(c=(-0.4,-0.7,0,0))$

\begin{tabular}{llr}
\hline$m=(0,0,0,0)$ & $m=(0,-0.12,0,0)$ & $m=(-0.12,-0.12,0,0)$ \\
\hline M sets & & \\
$(-0.57684,-0.40887,0,0)$ & $(-0.58115,-0.44337,0,0)$ & $(-0.56646,-0.47350,0,0)$ \\
$(-0.22360,-0.71034,0,0)$ & $(-0.19274,-0.67856,0,0)$ & $(-0.21800,-0.63569,0,0)$ \\
$(-0.62879,-0.71053,0,0)$ & $(-0.57161,-0.72858,0,0)$ & $(-0.52023,-0.69024,0,0)$ \\
$(-0.04451,-0.01817,0,0)$ & $(-0.27495,-0.11087,0,0)$ & $(-0.36281,-0.27713,0,0)$ \\
$(-0.40000,-0.70000,0,0)$ & $(-0.39922,-0.70190,0,0)$ & $(-0.38267,-0.69791,0,0)$ \\
$J$ sets & & $(-0.56646,-0.47350,0,0)$ \\
$(-0.57684,-0.40887,0,0)$ & $(-0.58115,-0.44337,0,0)$ & $(-0.21800,-0.63569,0,0)$ \\
$(-0.22360,-0.71034,0,0)$ & $(-0.19274,-0.67856,0,0)$ & $(-0.52023,-0.69024,0,0)$ \\
$(-0.62879,-0.71053,0,0)$ & $(-0.57161,-0.72858,0,0)$ & $(-0.36281,-0.27713,0,0)$ \\
$(-0.04451,-0.01817,0,0)$ & $(-0.27495,-0.11087,0,0)$ & $(-0.38267,-0.69791,0,0)$ \\
$(-0.40000,-0.70000,0,0)$ & $(-0.39922,-0.70190,0,0)$ & \\
\hline
\end{tabular}

so sensitive to the multiplicative noises, which indicate the periodicities of the $\mathrm{J}$ sets remain relatively stable. Different from the additive noise perturbations as discussed in Sect. 3.1, the multiplicative noises affect the escaping speed of the points. As a result, the images of the $\mathrm{M}$ sets shrink and rotate, the periodicity and the topology structures of the J sets changing correspondingly. Figure 9 shows the 3-D projections of the quaternion $\mathbf{J}$ sets with multiplicative noise perturbations.

\subsection{The stable periodic points of the quaternion $\mathrm{M}-\mathrm{J}$ sets}

As the additive noise disturbance we discussed, we also calculate the stable periodic points of multiplicative noise-perturbed $\mathrm{M}$ sets and $\mathrm{J}$ sets. Table 2 shows that the $\mathrm{M}$ sets and $\mathrm{J}$ sets under multiplicative noise disturbances have the same stable periodic points.

\section{Conclusions}

In this paper, the additive and dynamical noiseperturbed $\mathrm{M}-\mathrm{J}$ sets are discussed, the generalized $\mathrm{M}-\mathrm{J}$ sets in the quaternion space are constructed, and the two-dimensional slices of the sets are presented utilizing the cycle detecting method. The experimental results show that in the evolution process of the $\mathrm{M}$ sets dynamical system additive noise disturbs the directions and positions of the periodic regions and multiplicative noise disturbs the speed for escaping and iteration, which corresponds to the changes of the $\mathrm{M}$ sets in spatial region and time space, respectively. As the illustrated catalog of the $\mathrm{M}$ sets, the $\mathrm{J}$ sets also change with the spatial variations of the $\mathrm{M}$ sets.

We arrive at following conclusions:

(1) When the M sets are perturbed by the additive dynamic noise $m$, their topology structures remain stable, but the whole sets move $|m|$ distance along with the direction of $-m$. 
(2) The two arbitrary $\mathbf{J}$ sets can be converted to each other when they are perturbed by additive dynamic noise.

(3) When the M sets are perturbed by the multiplicative dynamic noise $k\left(k_{0}, k_{1}, k_{2}, k_{3}\right)$, taking the slice projections on the real axis $\mathbf{r}$ and image axis i for example, if both $k_{0}$ and $k_{1}$ are greater than -1 or less than -1 , the 1 -cycle region retains cardioid shape; if $k_{1}=k_{2}$, the whole sets shrink with certain proportion; the areas of the M sets become larger with the value of $k_{0}$ closer to -1 ; if both $k_{0}$ and $k_{1}$ are less than -1 , the $\mathrm{M}$ sets flip with 90 degrees.

(4) The points in the J sets escape faster under the perturbation of multiplicative dynamic noise, while the periodicity of the $\mathbf{J}$ sets retains relatively stable.

(5) The $\mathrm{M}$ sets and $\mathrm{J}$ sets still share the same stable points under both the additive and multiplicative noise perturbations.

Although the study is focused on basic additive and multiplicative dynamic noise models, the research methods we present can also be applied to other noise models. The future work can be extended to the research of the dynamic systems with sinusoidal noise and time-delay perturbations.

Acknowledgments This research is supported by the National Natural Science Foundation of China (Nos. 61103147, 61272373, 61272523), Trans-Century Training Program Foundation for the Talents by the Ministry of Education of China (NCET-13-0084), the National Key Project of Science and Technology of China (No. 2011ZX05039-003-4), Scientific Research Fund of Liaoning Provincial Education Department (No. L2014025) and the Fundamental Research Funds for the Central Universities (No. DUT15QY33).

Open Access This article is distributed under the terms of the Creative Commons Attribution 4.0 International License (http:// creativecommons.org/licenses/by/4.0/), which permits unrestricted use, distribution, and reproduction in any medium, provided you give appropriate credit to the original author(s) and the source, provide a link to the Creative Commons license, and indicate if changes were made.

\section{Appendix 1: Quaternions in terms of Pauli matrices and the associated quadratic map [28]}

The $2 \times 2$ Pauli matrices are defined by $\sigma=$ $\left(\sigma_{1}, \sigma_{2}, \sigma_{3}\right)$, where $\sigma_{1}=\left(\begin{array}{ll}0 & 1 \\ 1 & 0\end{array}\right), \quad \sigma_{2}=\left(\begin{array}{cc}0 & -\mathbf{i} \\ \mathbf{i} & 0\end{array}\right), \quad \sigma_{3}=\left(\begin{array}{cc}1 & 0 \\ 0 & -1\end{array}\right)$,

and the set is completed by the $2 \times 2$ unit matrices

$\mathrm{I}=\left(\begin{array}{ll}1 & 0 \\ 0 & 1\end{array}\right)$

The following properties of $\sigma$ are easily verified:

$\sigma_{l}^{2}=\mathrm{I} \quad l=1,2,3$,

$\sigma_{k} \sigma_{l}-\sigma_{l} \sigma_{k}=2 \mathbf{i} \in{ }_{k l m} \sigma_{m} l \neq k$

(Einstein's summation convention)

where $\epsilon_{k l m}$ is the totally antisymmetric Kronecker symbol.

Further

$\sigma_{l} \sigma_{k}+\sigma_{k} \sigma_{l}=0$,

Given two vectors $A$ and $B$, Eqs. (6.1)-(6.4) imply that

$(\sigma . A)(\sigma . B)=A . B+i \sigma . A \times B$.

In particular

$(\sigma . P)^{2}=P^{2}$

We define a quaternion $\{S, X, Y, Z\}=\{S, V\}$

by

$$
\begin{aligned}
Q & =S I+\mathbf{i} \sigma . V \\
& =\left[\begin{array}{cc}
S+\mathbf{i} Z & \mathbf{i}(X-\mathbf{i} Y) \\
\mathbf{i}(X+\mathbf{i} Y) & S-\mathbf{i} Z
\end{array}\right]
\end{aligned}
$$

The following Q properties of are easily established

$\|Q\|=S^{2}+V^{2}$

The eigenvalues $\lambda 1$ and $\lambda 2$ of $Q$ are given by

$\lambda_{2}=X \pm \mathbf{i}\|V\|$.

Given the equation

$\mathbb{R}^{2}=Q$,

we can define

$\mathbb{R}= \pm Q^{1 / 2}$,

where

$$
\begin{aligned}
Q^{1 / 2}= & {\left[\frac{S+\left(S^{2}+V^{2}\right)^{1 / 2}}{2}\right]^{1 / 2} } \\
& +\frac{\mathbf{i} \sigma . \mathrm{V}}{\left[S+\left(S^{2}+V^{2}\right)^{1 / 2}\right]^{1 / 2}},
\end{aligned}
$$




$$
\begin{aligned}
= & {\left[\frac{S+\left(S^{2}+V^{2}\right)^{1 / 2}}{2}\right]^{1 / 2} } \\
& +\frac{\mathbf{i} \sigma \cdot V}{|V|}\left[\frac{\left(S^{2}+V^{2}\right)^{1 / 2}-S}{2}\right]^{1 / 2}
\end{aligned}
$$

Following the analogy of the complex quadratic map[13], we introduce the quaternionic quadratic map

$Q \rightarrow Q^{2}+\mathbb{C}$

or

$Q_{n+1}=Q_{n}^{2}+\mathbb{C}$.

Using the algebra of the Pauli matrices, we obtain

$S_{n+1}=S_{n}^{2}-V_{n}^{2}+a$,

and

$V_{n+1}=2 S_{n} V_{n}+K$

or the equivalent set

$$
\begin{aligned}
S_{n+1} & =S_{n}^{2}-\left(X_{n}^{2}+Y_{n}^{2}+Z_{n}^{2}\right)+a, \\
Y_{n+1} & =2 S_{n} X_{n}+b, \\
Z_{n+1} & =2 S_{n} Y_{n}+c, \\
W_{n+1} & =2 S_{n} Z_{n}+d .
\end{aligned}
$$

\section{Appendix 2: Stability region of the 1-cycle of quaternion map $f: z \leftarrow z^{3}+c$ with additive dynamic noise}

Let $Q$ define the fixed point of the quaternion $\mathrm{M}$ set on the mapping $z \leftarrow z^{3}+c$, and then under the additive noise perturbation, it satisfies

$Q_{n+1}=Q_{n}^{3}+c+m$.

Then $Q_{n+1}$ can be given by

$$
\begin{aligned}
& S_{n+1}=S_{n}\left(S_{n}^{2}-3 V_{n}^{2}\right)+a+m_{1}, \\
& V_{n+1}=\left(3 S_{n}^{2}-V_{n}^{2}\right) V_{n}+K+m^{\prime} .
\end{aligned}
$$

Since $Q$ is the fixed point, the components $S$ and $V$ satisfy

$$
\begin{aligned}
& S=S\left(S^{2}-3 V^{2}\right)+a+m_{1}, \\
& V=\left(3 S^{2}-V^{2}\right) V+K+m^{\prime} .
\end{aligned}
$$

Introducing the perturbation of $Q$ :

$$
\begin{aligned}
S & =S_{n}+\delta S_{n}, \\
V & =V_{n}+\delta V_{n} .
\end{aligned}
$$

Then using Eqs. (7.2) and (7.3), it can be calculated that

$$
\begin{aligned}
\delta S_{n+1} & =S-S_{n+1} \\
& =S\left(S^{2}-3 V^{2}\right)-S_{n}\left(S_{n}^{2}-3 V_{n}^{2}\right),
\end{aligned}
$$

which can be simplified to

$$
\begin{aligned}
\delta S_{n+1}= & 3\left(S^{2}-V^{2}\right) \delta S_{n}-6 S V \cdot \delta V_{n} \\
& +O\left(\delta S_{n}^{2}, \delta V_{n}^{2}\right),
\end{aligned}
$$

where $O$ denotes "of the order of" [using Eq. (7.4)]. Similarly, we can get

$$
\begin{aligned}
\delta V_{n+1} & =V-V_{n+1} \\
& =\left(3 S^{2}-V^{2}\right) V-\left(3 S_{n}^{2}-V_{n}^{2}\right) V_{n},
\end{aligned}
$$

which can be simplified to

$$
\begin{aligned}
\delta V_{n+1}= & 6 S V \cdot \delta S_{n}+3\left(S^{2}-V^{2}\right) \delta V_{n} \\
& +O\left(\delta S_{n}^{2}, \delta V_{n}^{2}\right) .
\end{aligned}
$$

Now we expand $\delta V_{n}$ in terms of the orthogonal triplet $\left(K^{\prime}, I, J\right)$, where $K^{\prime}=K+m^{\prime}$, so that $\delta V_{n}=$ $\delta \alpha_{n} K^{\prime}+\delta \beta_{n} I+\delta \gamma_{n} J$, which reduce a $4 \times 4$ system to a $2 \times 2$ one. Ignoring terms of second order, Eq. (7.6) becomes

$$
\begin{aligned}
\delta S_{n+1}= & 3\left(S^{2}-V^{2}\right) \delta S_{n} \\
& -6 S V \cdot\left(\delta \alpha_{n} K^{\prime}\right),
\end{aligned}
$$

where $V=\frac{K^{\prime}}{1-3 S^{2}+V^{2}}$ [from Eq. (7.3)].

And Eq. (7.8) becomes

$$
\begin{aligned}
\delta V_{n+1}= & \frac{6 S K^{\prime}}{1-3 S^{2}+V^{2}} \cdot \delta S_{n} \\
& +3\left(S^{2}-V^{2}\right)\left(\delta \alpha_{n} K^{\prime}\right) .
\end{aligned}
$$

From Eqs. (7.9) and (7.10), we can get

$$
\left(\begin{array}{l}
\delta S_{n+1} \\
\delta \alpha_{n+1}
\end{array}\right)=J\left(\begin{array}{l}
\delta S_{n} \\
\delta \alpha_{n}
\end{array}\right)
$$

where $J=\left[\begin{array}{cc}3\left(S^{2}-V^{2}\right) & -6 S V \cdot K^{\prime} \\ \frac{6 S}{1-3 S^{2}+V^{2}} & 3\left(S^{2}-V^{2}\right)\end{array}\right]$. The eigenvalues of $J$ are given by

$\lambda=3\left(S^{2}-V^{2}\right) \pm 6 \mathbf{i} \sigma \cdot S V$.

Noting that $|\lambda|<1$, the boundary of the region of the stability can be determined by the condition as follows, $S^{2}+V^{2}=\frac{1}{3}$.

And $S$ and $V$ may be parameterized by

$$
S=\frac{\sqrt{3}}{3} \cos \theta, V=\frac{\sqrt{3}}{3} \sin \theta \cdot n(0 \leq \theta<2 \pi)
$$

where $n$ is an arbitrary unit vector.

Thus the boundary of the region of the fixed points is given by 


$$
\begin{aligned}
& a=\frac{\sqrt{3}}{3} \cos \theta-\frac{\sqrt{3}}{9} \cos 3 \theta-m_{1}, \\
& K=\left(\frac{\sqrt{3}}{3} \sin \theta-\frac{\sqrt{3}}{9} \sin 3 \theta\right) n-m^{\prime}(0 \leq \theta<2 \pi) .
\end{aligned}
$$

\section{Appendix 3: Stability region of 1-cycle of quaternion $M$ set with multiplicative dynamic noise on the map $z \leftarrow z^{3}+c$}

Let $Q$ define the fixed point of the quaternion $\mathrm{M}$ set with multiplicative dynamic noise on the map $z \leftarrow z^{3}+c$, and then according to the definition in Eq. (2.2), the components of $Q_{n+1}$ can be given by

$$
\begin{aligned}
S_{n+1} & =\left(1+k_{1}\right) h^{3} \cos 3 \theta+a \\
& =S_{n}\left(1+k_{1}\right)\left(S_{n}^{2}-3 V_{n}^{2}\right)+a, \\
V_{n+1} & =\left(1+k_{2}\right) h^{3} \sin 3 \theta+K \\
& =V_{n}\left(1+k_{2}\right)\left(3 S_{n}^{2}-V_{n}^{2}\right)+K,
\end{aligned}
$$

And the components $S$ and $V$ of $Q$ satisfy

$$
\begin{aligned}
& S=S\left(1+k_{1}\right)\left(S^{2}-3 V^{2}\right)+a, \\
& V=V\left(1+k_{2}\right)\left(3 S^{2}-V^{2}\right)+K .
\end{aligned}
$$

Then it can be calculated that

$$
\begin{aligned}
& \delta S_{n+1}=S-S_{n+1} \\
& \quad=3\left(1+k_{1}\right)\left(S^{2}-V^{2}\right) \delta S_{n}-6\left(1+k_{1}\right) S V \cdot \delta V_{n}, \\
& \delta V_{n+1}=V-V_{n+1} \\
& \quad=6\left(1+k_{2}\right) S V \cdot \delta S_{n}+3\left(1+k_{2}\right)\left(S^{2}-V^{2}\right) \delta V_{n},
\end{aligned}
$$

where terms of second order and higher have been ignored.

Now we expand $\delta V_{n}$ in terms of the orthogonal triplet $(K, I, J)$, and Eq. (8.3) becomes

$$
\begin{aligned}
\delta S_{n+1}= & 3\left(1+k_{1}\right)\left(S^{2}-V^{2}\right) \delta S_{n} \\
& -6\left(1+k_{1}\right) S V \cdot\left(\delta \alpha_{n} K\right),
\end{aligned}
$$

where $V=\frac{K}{1-\left(1+k_{2}\right)\left(3 S^{2}-V^{2}\right)}$ [from Eq. (8.2b)].

Equation (8.4) becomes

$$
\begin{aligned}
\delta V_{n+2}= & \frac{6\left(1+k_{2}\right) S}{1-\left(1+k_{2}\right)\left(3 S^{2}-V^{2}\right)} K \cdot \delta S_{n} \\
& +3\left(1+k_{2}\right)\left(S^{2}-V^{2}\right)\left(\delta \alpha_{n} K\right)
\end{aligned}
$$

From Eqs. (8.5) and (8.6), we can get

$$
\left(\begin{array}{l}
\delta S_{n+2} \\
\delta a_{n+2}
\end{array}\right)=J\left(\begin{array}{l}
\delta S_{n} \\
\delta a_{n}
\end{array}\right)
$$

where $J=\left[\begin{array}{cc}3\left(1+k_{1}\right)\left(S^{2}-V^{2}\right) & -6\left(1+k_{1}\right) S V \\ \frac{6\left(1+k_{2}\right) S}{1-\left(1+k_{2}\right)\left(3 S^{2}-V^{2}\right)} & K 3\left(1+k_{2}\right)\left(S^{2}-V^{2}\right)\end{array}\right]$. The eigenvalues of $J$ are given by

$$
\begin{aligned}
\lambda= & \frac{3}{2}(p+q)\left(S^{2}-V^{2}\right) \pm \frac{3}{2} \mathbf{i} \sigma \cdot\left[4 p q\left(S^{2}+V^{2}\right)^{2}\right. \\
& \left.-(p+q)^{2}\left(S^{2}-V^{2}\right)^{2}\right]^{1 / 2}
\end{aligned}
$$

where $p=1+k_{1}, q=1+k_{2}$. Noting that $|\lambda|<1$, the boundary of the region of the stability can be determined by the condition as follows,

$S^{2}+V^{2}=\frac{1}{3 \sqrt{p q}}$.

And $S$ and $V$ may be parameterized by

$$
\begin{aligned}
S & =\left(\frac{1}{9 p q}\right)^{\frac{1}{4}} \cos \theta, \\
V & =\left(\frac{1}{9 p q}\right)^{\frac{1}{4}} \sin \theta \cdot n(0 \leq \theta<2 \pi)
\end{aligned}
$$

where $n$ is an arbitrary unit vector.

Thus the boundary of the region of the fixed points is given by

$$
\begin{aligned}
a= & \left(\frac{1}{9 p q}\right)^{\frac{1}{4}} \cos \theta-\alpha\left(\frac{1}{9 p q}\right)^{\frac{3}{4}} \cos 3 \theta, \\
K= & {\left[\left(\frac{1}{9 p q}\right)^{\frac{1}{4}} \sin \theta-\beta\left(\frac{1}{9 p q}\right)^{\frac{3}{4}} \sin 3 \theta\right] } \\
& n(0 \leq \theta<2 \pi) .
\end{aligned}
$$

\section{References}

1. Norton, A.: Julia sets in the quaternions. Comput. Gr. 13(2), 267-278 (1989)

2. Nakane, S.: Dynamics of a family of quadratic maps in the quaternion space. Int. J. Bifurc Chaos 15(8), 2535-2543 (2005)

3. Halayka, S.: Some visually interesting non-standard quaternion fractal sets. Chaos Solitons Fract. 41(5), 2842-2846 (2009)

4. Chen, N., Li, Z., Jin, Y.: Visual presentation of dynamic systems with hyperbolic planar symmetry. Chaos Solitons Fract. 40(2), 621-634 (2009)

5. Sun, Y.Y., Wang, X.Y.: Quaternion $M$ set with none zero critical points. Fractals 17(4), 427-439 (2009) 
6. Longtin, A.: Effects of noise on nonlinear dynamics. In:Beuter, A., Glass, L., Mackey, M.C., Titcombe, M.S. (eds.) Nonlinear Dynamics in Physiology and Medicine. New York: Springer, pp. 149-189 (2003)

7. Oritz, N.: Dynamical systems with time delay. (Doctor Thesis) Louisiana State University, USA (2005)

8. Argyris, J., Andreadis, I., Karakasidis, T.E.: On perturbations of the Mandelbrot map. Chaos Solitons Fract. 11(7), 1131-1136 (2000)

9. Argyris, J., Karakasidis, T.E., Andreadis, I.: On the Julia set of the perturbed Mandelbrot map. Chaos Solitons Fract. 11(13), 2067-2073 (2000)

10. Argyris, J., Karakasidis, T.E., Andreadis, I.: On the Julia set of a noise-perturbed Mandelbrot map. Chaos Solitons Fract. 13(2), 245-252 (2002)

11. Andreadis, I., Karakasidis, T.E.: On a topological closeness of perturbed Julia sets. Appl. Math. Comput. 217(6), 28832890 (2010)

12. Andreadis, I., Karakasidis, T.E.: On a topological closeness of perturbed Mandelbrot sets. Appl. Math. Comput. 215(10), 3674-3683 (2010)

13. Andreadis, I., Karakasidis, T.E.: On probabilistic Mandelbrot maps. Chaos Solitons Fract. 42(3), 1577-1583 (2009)

14. Negi, A., Rani, M.: A new approach to dynamic noise on superior Mandelbrot set. Choas Solitons Fract. 36(4), 10891096 (2008)

15. Rani, M., Agarwal, R.: Effect of stochastic noise on superior Julia sets. J. Math. Imaging Vis. 36(1), 63-68 (2010)

16. Agarwal, R., Agarwal, V.: Dynamic noise perturbed generalized superior Mandelbrot sets. Nonlinear Dyn. 67(3), 1883-1891 (2012)

17. Rani, M., Agarwal, R.: General noise-perturbed superior Julia sets. J. Comput. Nonlinear Dyn. 8(2), 024501 (2013)
18. Wang, X.Y., Chang, P.J., Gu, N.N.: Additive perturbed generalizedMandelbrot-Julia sets. Appl. Math. Comput. 189(1), 754-765 (2007)

19. Wang, X.Y., Jia, R.H., Sun, Y.Y.: The generalized Julia set perturbed by composing additive and multiplicative noises. Discrete Dyn. Nat. Soc. (2009). http://dx.doi.org/10.1155/ 2009/781976

20. Sun, Y.Y., Wang, X.Y.: General quaternion M sets with noise perturbations. In: 2009 International Workshop on ChaosFractals Theories and Applications (IWCFTA 2009), pp. 417-421 (2009)

21. Eliazar, I.: Intrinsic fractality of classic shot noise. Phys. Rev. E 77(6), 061103 (2008)

22. Wang, X.Y., Song, W.J.: The generalized M-J sets for bicomplex numbers. Nonlinear Dyn. 72(1-2), 17-26 (2013)

23. Liu, P.: Control and synchronization of Mandelbrot sets in coupled map lattice. Nonlinear Dyn. 73(1-2), 299-310 (2013)

24. Danca, M.F., Bourke, P., Romera, M.: Graphical exploration of the connectivity sets of alternated Julia sets. Nonlinear Dyn. 73(1-2), 1155-1163 (2013)

25. Argyris, J., Andreadis, I., Pavlos, G.: The influence of noise on the correlation dimension of chaotic attractors. Chaos Solitons Fract. 9(3), 343-361 (1998)

26. Crutchfield, J.P., Farmer, J.D., Huberman, B.A.: Fluctuations and simple chaotic dynamics. Phys. Rep. 92(2), 45-82 (1982)

27. Wang, X.Y., Sun, Y.Y.: The general quaternionic M-J sets on the mapping $\mathrm{z} \leftarrow \mathrm{z} \wedge \mathrm{a}+\mathrm{c}(\mathrm{a} \in \mathrm{N})$. Comput. Math. Appl. 53(11), 1718-1732 (2007)

28. Gomatam, J., Doyle, J., Steves, B., et al.: Generalization of the Mandelbrot set: quaternionic quadratic maps. Chaos Solitons Fract. 5(6), 971-986 (1995) 